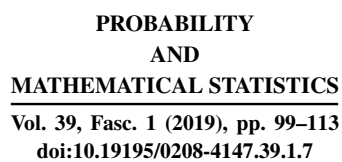

\title{
OCCUPATION TIME PROBLEM FOR MULTIFRACTIONAL BROWNIAN MOTION
}

BY

\author{
MOHAMED A I T OUAHRA (OUJDA), RABY GUERBAZ (CASABLANCA), \\ HANAE OUAHHABI (AL AIN), AND AISSA SGHIR (OUJDA)
}

Abstract. In this paper, by using a Fourier analytic approach, we investigate sample path properties of the fractional derivatives of multifractional Brownian motion local times. We also show that those additive functionals satisfy a property of local asymptotic self-similarity. As a consequence, we derive some local limit theorems for the occupation time of multifractional Brownian motion in the space of continuous functions.

2010 AMS Mathematics Subject Classification: Primary: 60F25, 60J55; Secondary: 60G22.

Key words and phrases: Local time, local asymptotic self-similarity, limit theorem, fractional Brownian motion, multifractional Brownian motion, fractional derivative.

\section{INTRODUCTION}

A fractional Brownian motion (fBm for short) $B^{H}=\left\{B^{H}(t), t \geqslant 0\right\}$ with Hurst index $H \in(0,1)$ is a real-valued, centered Gaussian process with covariance function given by

$$
\mathbb{E}\left(B^{H}(t) B^{H}(s)\right)=\frac{1}{2}\left(s^{2 H}+t^{2 H}-|s-t|^{2 H}\right) \quad \text { for all } s, t \geqslant 0 .
$$

Several properties of fBm such as self-similarity and the stationarity of increments make it important in theory and also a useful model in various applications, such as in telecommunication, finance, image analysis among others. Note that in the case $H=\frac{1}{2}$, we retrieve the well-known Brownian motion. The fact that the Hurst parameter $H$ is independent of time $t$ makes the Hölder regularity of $\mathrm{fBm}$ constant along the paths. This property restricts its applications when modelling phenomena whose regularity evolves in time, such as Internet traffic and some highly textured images with strong global organization; see, for example, Lévy-Véhel [115] and Pesquet-Popescu and Lévy-Véhel [18]. 
The multifractional Brownian motion ( $\mathrm{mBm}$ for short) was introduced as a natural extension of $\mathrm{fBm}$ to overcome this limitation. The basic idea is to replace the Hurst parameter $H$ by a function $H(t):[0, \infty) \rightarrow[a, b] \subset(0,1)$ which is a Hölder continuous function of exponent $\beta>0$, i.e., there exists a finite positive constant $C$ such that

$$
|H(t)-H(s)| \leqslant C|t-s|^{\beta} \quad \text { for all } s, t \geqslant 0 .
$$

1. Moving average representation of mBm (Lévy-Véhel and Peltier [16]):

$$
\begin{aligned}
\widetilde{B}^{H(t)}(t)= & \frac{1}{\Gamma\left(H(t)+\frac{1}{2}\right)}\left(\int_{-\infty}^{0}\left[(t-u)^{H(t)-1 / 2}-(-u)^{H(t)-1 / 2}\right] W(d u)\right. \\
& \left.+\int_{0}^{t}(t-u)^{H(t)-1 / 2} W(d u)\right), \quad t \geqslant 0
\end{aligned}
$$

where $W$ is a standard Brownian motion defined on $\mathbb{R}$.

2. Harmonizable representation of $\mathrm{mBm}$ (Benassi et al. [4]):

$$
\widehat{B}^{H(t)}(t)=\int_{\mathbb{R}} \frac{e^{i t \xi}-1}{|\xi|^{H(t)+1 / 2}} \widehat{W}(d \xi), \quad t \geqslant 0,
$$

where $\widehat{W}(\xi)$ is the Fourier transform of the series representation of white noise with respect to an orthonormal basis of $L^{2}(\mathbb{R})$.

In the sequel, the notation $B:=(B(t), t \geqslant 0)$ means that both previous representations of $\mathrm{mBm}$ may be chosen.

When $H(\cdot)$ varies with time, the $\mathrm{mBm}$ is no longer self-similar, even though it is seen as an intricacy; Lévy-Véhel and Peltier [16] proved that if $H(\cdot)$ satisfies the assumption

$$
\left(\mathcal{H}_{\beta}\right):\left\{H(\cdot) \text { is } \beta \text {-Hölder continuous and } \sup _{t \in \mathbb{R}^{+}} H(t)<\beta\right\},
$$

then the $\mathrm{mBm}$ has a property called the local asymptotic self-similarity (LASS in short) defined as follows:

$$
\lim _{\rho \rightarrow 0^{+}} \operatorname{law}\left\{\frac{B(t+\rho u)-B(t)}{\rho^{H(t)}}, u \in \mathbb{R}\right\}=\operatorname{law}\left\{B^{H(t)}(u), u \in \mathbb{R}\right\},
$$

where $B^{H(t)}$ is an $\mathrm{fBm}$ with the Hurst parameter $H(t)$.

By using the concept of local nondeterminism and the assumption $\left(\mathcal{H}_{\beta}\right)$, Boufoussi et al. [6] proved the existence of a jointly continuous local time of $\mathrm{mBm}$ and studied its Hölder regularity in time and space. In Boufoussi et al. [ $[$ ], the 
authors proved that the local time of $\mathrm{mBm}$ has a kind of local asymptotic selfsimilarity (LASS) property. Through this result, they obtained some local limit theorems corresponding to the $\mathrm{mBm}$ (first order limit theorems).

The aim of this work is to establish some local limit theorems for the occupation time of $\mathrm{mBm}$. To prove our main results we are led to study the regularities of the fractional derivative of local time of $\mathrm{mBm}$, by using a representation based on Fourier analytic approach.

The rest of this paper is organized as follows. In Section 2, we give some basic facts about local times. In Section 3, we prove some Hölder regularities of the fractional derivative of local time of $\mathrm{mBm}$. Section 4 is devoted to the asymptotic results: Subsection 4.1 is concerned with the LASS property of those additive functionals, and in Subsection 4.2, some local limit theorems for the occupation time of $\mathrm{mBm}$ are studied.

Most of the estimates in the sequel contain unspecified finite positive constants. We use the same symbol for these constants, even when they vary from one line to the next.

\section{LOCAL TIMES}

In this section we recall the definition and some properties of local times of Gaussian processes and a result on $\mathrm{mBm}$ that will be needed in the sequel.

Let $\left(X(t), t \in \mathbb{R}^{+}\right)$be a separable random process with Borel sample function. The occupation measure of $X$ is defined as follows:

$$
\mu(A, B)=\lambda(\{s \in A, X(s) \in B\}) \quad \forall A \in \mathcal{B}\left(\mathbb{R}^{+}\right), \forall B \in \mathcal{B}(\mathbb{R}),
$$

where $\lambda$ is the Lebesgue measure on $\mathbb{R}^{+}$. If $\mu(A, \cdot)$ is absolutely continuous with respect to the Lebesgue measure on $\mathbb{R}$, we say that $X$ has local time on $A$ and define the local time $L(A, \cdot)$ as the Radon-Nikodym derivative of $\mu(A, \cdot)$. Sometimes, we write $L_{t}^{x}$ instead of $L([0, t], x)$.

The following property of local time is called the occupation density formula:

For every $t \in \mathbb{R}^{+}$and every measurable function $f: \mathbb{R} \mapsto \mathbb{R}^{+}$,

$$
\int_{0}^{t} f\left(X_{s}\right) d s=\int_{\mathbb{R}} f(x) L_{t}^{x} d x
$$

The well-known Fourier analytic approach introduced by Berman [5] states that, for a fixed sample function at fixed $t$, the Fourier transform on $x$ of $L_{t}^{x}$ is the function

$$
F(u)=\int_{\mathbb{R}} e^{i u x} L_{t}^{x} d x .
$$

Using the occupation density formula and the inverse Fourier transform of this 
function, we have

$$
L_{t}^{x}=\frac{1}{2 \pi} \int_{-\infty}^{+\infty}\left(\int_{0}^{t} e^{i u(X(s)-x)} d s\right) d u .
$$

The following result due to Boufoussi et al. ([6], Lemma 3.3) will extensively be used in the sequel.

LEMMA 2.1. Under the assumption $\left(\mathcal{H}_{\beta}\right)$, for any even integer $m \geqslant 2$, there exist positive and finite constants $C_{m}>0$ and $\delta>0$ such that, for any $t, s \geqslant 0$ with $|t-s|<\delta$, any $x, y \in \mathbb{R}$ and any $0<\xi<\min (1,(1-\lambda) /(2 \lambda))$, where $\lambda=\sup _{u \in[s, t]} H(u)$, we have

$$
\begin{gathered}
\mathbb{E}\left[L_{t}^{x}-L_{s}^{x}\right]^{m} \leqslant C_{m} \frac{|t-s|^{m(1-\lambda)}}{\Gamma(1+m(1-\lambda))}, \\
\mathbb{E}\left[L_{t}^{y}-L_{s}^{y}-L_{t}^{x}+L_{s}^{x}\right]^{m} \leqslant C_{m}|y-x|^{\xi m} \frac{|t-s|^{m(1-\lambda(1+\xi))}}{\Gamma(1+m(1-\lambda(1+\xi)))} .
\end{gathered}
$$

REMARK 2.1. Estimates similar to ([2.2) and ([2.3) were proved for the multifractional Brownian sheet by Meerschaert et al. [17], Lemmas 3.5 and 3.7.

\section{FRACTIONAL DERIVATIVE OF LOCAL TIME OF mBm}

The fractional derivatives have many uses, such as fractional integro-differentiation which has now become a significant topic in mathematical analysis. Fractional derivatives of local time have been discussed for physical purposes in the paper by Ezawa et al. [9]. For a complete survey on the fractional derivative we refer the reader to Hardy and Littlewood [13] and the book by Samko et al. [19].

DEFinition 3.1. Let $0<\theta<1$ and $f: \mathbb{R} \rightarrow \mathbb{R}$ be a function that belongs to $\mathcal{C}^{\theta} \cap L^{1}(\mathbb{R})$, where $\mathcal{C}^{\theta}$ is the space of locally $\theta$-Hölder continuous functions on $\mathbb{R}$. For $0<\gamma<\theta$, we define $D_{ \pm}^{\gamma} f$ by

$$
D_{ \pm}^{\gamma} f(x):=\frac{\gamma}{\Gamma(1-\gamma)} \int_{0}^{+\infty} \frac{f(x)-f(x \mp y)}{y^{1+\gamma}} d y
$$

The operators $D_{+}^{\gamma}$ and $D_{-}^{\gamma}$ are called, respectively, right-sided and left-sided Marchaud fractional derivatives of order $\gamma$. We put $D^{\gamma}:=D_{+}^{\gamma}-D_{-}^{\gamma}$.

REMARK 3.1. 1. $D_{+}^{\gamma}$ and $D_{-}^{\gamma}$ satisfy the switching identity:

$$
\int_{\mathbb{R}} f(x) D_{-}^{\gamma} g(x) d x=\int_{\mathbb{R}} g(x) D_{+}^{\gamma} f(x) d x
$$

for any $f, g \in \mathcal{C}^{\theta}(\mathbb{R}) \cap L^{1}(\mathbb{R})$ and $0<\gamma<\theta$. 
2. $D_{ \pm}^{\gamma} f$ is $(\theta-\gamma)$-Hölder continuous whenever $f$ is $\theta$-Hölder continuous for any $0<\gamma<\theta$.

3. For $h: \mathbb{R} \rightarrow \mathbb{R}$ and $a>0$, we denote by $h_{a}$ the function $x \rightarrow h(a x)$; then

$$
D_{ \pm}^{\gamma}\left(h_{a}\right)=a^{\gamma}\left(D_{ \pm}^{\gamma} h\right)_{a} \quad \text { for all } \gamma>0 .
$$

4. The fractional derivatives of the local time are particular continuous additive functionals of zero energy in the sense of Fukushima [11].

Lemma 2.1 allows us to define the fractional derivative of order $\gamma$ of local time of $\mathrm{mBm}$ as follows:

$$
D^{\gamma} L(t, \cdot)(x):=\frac{\gamma}{\Gamma(1-\gamma)} \int_{0}^{\infty} \frac{L_{t}^{x+a}-L_{t}^{x-a}}{a^{1+\gamma}} d a \text { for all } 0<\gamma<\xi .
$$

Using (2.1), we get

$$
\begin{aligned}
& D^{\gamma} L(t, \cdot)(x) \\
& :=\frac{\gamma}{2 \pi \Gamma(1-\gamma)} \int_{0}^{+\infty} \int_{\mathbb{R}}^{t} \int_{0}^{t} \frac{e^{i u(B(s)-(x+a))}-e^{i u(B(s)-(x-a))}}{a^{1+\gamma}} d s d u d a \\
& \quad=\frac{\gamma}{2 \pi \Gamma(1-\gamma)} \int_{0}^{+\infty} \int_{\mathbb{R}}^{t} \int_{0}^{t} e^{i u B(s)}\left[e^{-i u(x+a)}-e^{-i u(x-a)}\right] \frac{1}{a^{1+\gamma}} d s d u d a .
\end{aligned}
$$

Here are the main results of this section.

THEOREM 3.1. Let $0<\gamma<\xi$ and $D \in\left\{D^{\gamma}, D_{ \pm}^{\gamma}\right\}$. Under the assumption $\left(\mathcal{H}_{\beta}\right)$, there exist finite and positive constants $C_{m}>0$ and $\delta>0$ such that for $\mathrm{ev}$ ery $t, s \geqslant 0$ with $|t-s|<\delta$, any $x \in \mathbb{R}$ and any $0<\xi<\min (1,(1-\lambda) /(2 \lambda))$, where $\lambda=\sup _{u \in[s, t]} H(u)$, we have

$$
\|D L(t, \cdot)(x)-D L(s, \cdot)(x)\|_{2 m} \leqslant C_{m}|t-s|^{1-\lambda(1+\gamma)},
$$

where $\|\cdot\|_{2 m}=\left[\mathbb{E}|\cdot|^{2 m}\right]^{1 /(2 m)}$.

Pro of. We will prove (3.2) for $D^{\gamma}$ since the other cases may be treated in the same way. For all integers $m \geqslant 1$, we have for any $b>0$

$$
\begin{aligned}
\left\|D^{\gamma} L_{t}(x)-D^{\gamma} L_{s}(x)\right\|_{2 m} & =\left\|\int_{0}^{+\infty} \frac{\gamma}{\Gamma(1-\gamma)} \frac{L_{t}^{x+u}-L_{t}^{x-u}-L_{s}^{x+u}+L_{s}^{x-u}}{u^{1+\gamma}} d u\right\|_{2 m} \\
& \leqslant C\left(I_{1}(b)+I_{2}(b)\right)
\end{aligned}
$$

where

$$
I_{1}(b):=\int_{0}^{b} \frac{\left\|L_{t}^{x+u}-L_{t}^{x-u}-L_{s}^{x+u}+L_{s}^{x-u}\right\|_{2 m}}{u^{1+\gamma}} d u
$$


and

$$
I_{2}(b):=\int_{b}^{+\infty} \frac{\left\|L_{t}^{x+u}-L_{t}^{x-u}-L_{s}^{x+u}+L_{s}^{x-u}\right\|_{2 m}}{u^{1+\gamma}} d u .
$$

We estimate $I_{1}(b)$ and $I_{2}(b)$ separately.

In view of $(\underline{2.3})$, we deduce

$$
I_{1}(b) \leqslant C_{m}|t-s|^{1-\lambda(1+\xi)} \int_{0}^{b} u^{\xi-\gamma-1} d u \leqslant C_{m}|t-s|^{1-\lambda(1+\xi)} b^{\xi-\gamma}
$$

Now, we deal with $I_{2}(b)$. We use (2.2) to conclude that

$$
I_{2}(b) \leqslant C_{m}|t-s|^{1-\lambda} \int_{b}^{+\infty} \frac{1}{u^{1+\gamma}} d u \leqslant C_{m}|t-s|^{1-\lambda} b^{-\gamma} .
$$

Finally, by choosing $b=|t-s|^{\lambda}$, we obtain the desired result.

THEOREM 3.2. Let $0<\gamma<\xi$ and $D \in\left\{D^{\gamma}, D_{ \pm}^{\gamma}\right\}$. Under the assumption $\left(\mathcal{H}_{\beta}\right)$, there exists a finite and positive constant $C_{m}>0$ such that, for every $t, s \geqslant 0$ with $|t-s|<\delta$, any $(x, y) \in \mathbb{R}^{2}$ and any $0<\xi<\min (1,(1-\lambda) /(2 \lambda))$, where $\lambda=\sup _{u \in[s, t]} H(u)$, we have

$$
\begin{aligned}
&\|D L(t, \cdot)(x)-D L(t, \cdot)(y)-D L(s, \cdot)(x)+D L(s, \cdot)(y)\|_{2 m} \\
& \leqslant C_{m}|t-s|^{1-\lambda(1+\xi)}|x-y|^{\xi-\gamma}
\end{aligned}
$$

REMARK 3.2. Similar results are obtained, respectively, by Ait Ouahra and Eddahbi [2] and Ait Ouahra [1] for the local time and the fractional derivative of local time of a symmetric stable process of index $1<\alpha \leqslant 2$. The key ingredient in their proofs was the Markov property of the symmetric stable process, which is not satisfied by the $\mathrm{mBm}$.

Proof of Theorem 3.2. For any integer $m \geqslant 1$, we have

$$
\begin{aligned}
& \|D L(t, \cdot)(x)-D L(t, \cdot)(y)-D L(s, \cdot)(x)+D L(s, \cdot)(y)\|_{2 m} \\
= & \left\|\int_{[0, \infty)} \frac{L_{t}^{x+u}-L_{s}^{x+u}-L_{t}^{x-u}+L_{s}^{x-u}-L_{t}^{y+u}+L_{s}^{y+u}+L_{t}^{y-u}-L_{s}^{y-u}}{u^{1+\gamma}} d u\right\|_{2 m} \\
\leqslant & C \int_{[0, \infty)}\left\|\frac{L_{t}^{x+u}-L_{s}^{x+u}-L_{t}^{x-u}+L_{s}^{x-u}-L_{t}^{y+u}+L_{s}^{y+u}+L_{t}^{y-u}-L_{s}^{y-u}}{u^{1+\gamma}}\right\|_{2 m} d u \\
\leqslant & C\left(J_{1}(b)+J_{2}(b)\right),
\end{aligned}
$$


where

$$
\begin{aligned}
& J_{1}(b) \\
:= & \int_{[0, b]}\left\|\frac{L_{t}^{x+u}-L_{s}^{x+u}-L_{t}^{x-u}+L_{s}^{x-u}-L_{t}^{y+u}+L_{s}^{y+u}+L_{t}^{y-u}-L_{s}^{y-u}}{u^{1+\gamma}}\right\|_{2 m} d u, \\
:= & \int_{[b,+\infty}\left\|\frac{L_{t}^{x+u}-L_{s}^{x+u}-L_{t}^{x-u}+L_{s}^{x-u}-L_{t}^{y+u}+L_{s}^{y+u}+L_{t}^{y-u}-L_{s}^{y-u}}{u^{1+\gamma}}\right\|_{2 m} d u
\end{aligned}
$$

for some $b>0$.

First, let us estimate $J_{1}(b)$. Combining the Minkowski inequality and (2.3), we obtain

$$
\begin{aligned}
& \left\|L_{t}^{x+u}-L_{s}^{x+u}-L_{t}^{x-u}+L_{s}^{x-u}-L_{t}^{y+u}+L_{s}^{y+u}+L_{t}^{y-u}-L_{s}^{y-u}\right\|_{2 m} \\
\leqslant & \left\|L_{t}^{x+u}-L_{s}^{x+u}-L_{t}^{x-u}+L_{s}^{x-u}\right\|_{2 m}+\left\|L_{t}^{y+u}-L_{s}^{y+u}-L_{t}^{y-u}+L_{s}^{y-u}\right\|_{2 m} \\
\leqslant & C_{m} \frac{|u|^{\xi}|t-s|^{(1-\lambda(1+\xi))}}{\Gamma(1+2 m(1-\lambda(1+\xi)))} .
\end{aligned}
$$

Therefore,

$$
\begin{aligned}
J_{1}(b) & \leqslant C_{m} \frac{|t-s|^{(1-\lambda(1+\xi))}}{\Gamma(1+2 m(1+\lambda(1+\xi)))} \int_{[0, b]} \frac{u^{\xi}}{u^{1+\gamma}} d u \\
& \leqslant C_{m} \frac{|t-s|^{(1-\lambda(1+\xi))}}{\Gamma(1+2 m(1-\lambda(1+\xi)))} b^{\xi-\gamma} .
\end{aligned}
$$

Now, we are going to estimate $J_{2}(b)$. Using the Minkowski inequality and decomposing otherwise, we obtain

$$
\begin{aligned}
& \left\|L_{t}^{x+u}-L_{s}^{x+u}-L_{t}^{x-u}+L_{s}^{x-u}-L_{t}^{y+u}+L_{s}^{y+u}+L_{t}^{y-u}-L_{s}^{y-u}\right\|_{2 m} \\
\leqslant & \left\|L_{t}^{x+u}-L_{s}^{x+u}-L_{t}^{y+u}+L_{s}^{y+u}\right\|_{2 m}+\left\|L_{t}^{x-u}-L_{s}^{x-u}-L_{t}^{y-u}+L_{s}^{y-u}\right\|_{2 m} \\
\leqslant & C_{m} \frac{|x-y|^{\xi}|t-s|^{(1-\lambda(1+\xi))}}{\Gamma(1+2 m(1-\lambda(1+\xi)))} .
\end{aligned}
$$

Therefore, $J_{2}(b)$ is dominated by

$$
b^{-\gamma} \cdot C_{m} \frac{|x-y|^{\xi}|t-s|^{(1-\lambda(1+\xi))}}{\Gamma(1+2 m(1-\lambda(1+\xi)))} .
$$


By choosing $b=|x-y|$, we deduce that

$$
\begin{aligned}
\|D L(t, \cdot)(x)-D L(t, \cdot)(y)-D L(s, \cdot)(x)+D L(s, \cdot)(y)\|_{2 m} & \\
& \leqslant C_{m}|t-s|^{(1-\lambda(1+\xi))}|x-y|^{\xi-\gamma},
\end{aligned}
$$

which completes the proof of the theorem.

\section{ASYMPTOTIC RESULTS}

It is well known that techniques for proving limit theorems related to selfsimilar processes use the self-similarity of their local times. It is natural to expect the same when dealing with processes satisfying the LASS property. The answer to the preceding question is affirmative in the case of the $\mathrm{mBm}$ and the result is given by the following lemma. (See Theorem 5.1 in Boufoussi et al. [⿴囗] ].)

In the sequel, we will use the following notation: $B^{H(t)}(t)=B(t)$ for the sake of simplicity.

LEMMA 4.1. Under the assumption $\left(\mathcal{H}_{\beta}\right)$, for any fixed $t_{0}$, the local time of $m B m$ is locally asymptotically self-similar with parameter $1-H\left(t_{0}\right)$, in the sense that, for every $x \in \mathbb{R}$, the family of processes $\left\{Y_{\rho}(t, x), t \in[0,1]\right\}_{\rho>0}$ defined by

$$
Y_{\rho}(t, x)=\frac{L\left(t_{0}+\rho t, \rho^{H\left(t_{0}\right)} x+B\left(t_{0}\right)\right)-L\left(t_{0}, \rho^{H\left(t_{0}\right)} x+B\left(t_{0}\right)\right)}{\rho^{1-H\left(t_{0}\right)}}
$$

converges in law to the local time $\{l(t, x), t \in[0,1]\}$ of the $f B m B^{H\left(t_{0}\right)}$ with Hurst parameter $H\left(t_{0}\right)$.

We will introduce the proof of this lemma in the Appendix, to clarify a passage in the estimate of $I_{1}^{\varepsilon, \rho}$ on page 864 in Boufoussi et al. [7].

REMARK 4.1. The previous result can also be obtained by using propositions (4.1) and (4.2) in Jolis and Viles [14], which allows us to identify the limit law as local time.

\subsection{LASS for the fractional derivative of local time of $\mathrm{mBm}$.}

Proposition 4.1. Under the assumption $\left(\mathcal{H}_{\beta}\right)$, for any fixed $t_{0}$, the fractional derivative of local time of $\mathrm{mBm}$ is LASS with parameter $1-(1+\gamma) H\left(t_{0}\right)$, in the sense that, for every $x \in \mathbb{R}$, the family of processes $\left\{D_{\rho}(t, x), t \in[0,1]\right\}_{\rho} \geqslant 0$ defined by

$$
D_{\rho}(t, x):=\frac{D^{\gamma} L\left(t_{0}+t \rho, \cdot\right)\left(\rho^{H\left(t_{0}\right)} x+B\left(t_{0}\right)\right)-D^{\gamma} L\left(t_{0}, \cdot\right)\left(\rho^{H\left(t_{0}\right)} x+B\left(t_{0}\right)\right)}{\rho^{1-H\left(t_{0}\right)(1+\gamma)}}
$$

converges in law to the fractional derivative $\left\{D^{\gamma} l_{t}(x), t \in[0,1]\right\}$ of local time of the $\mathrm{fBm} B^{H\left(t_{0}\right)}$. 
Pr o of. To prove the convergence in law, we proceed in two steps. First, we prove the tightness of the family $\left\{D_{\rho}(t, x), t \in[0,1]\right\}_{\rho \geqslant 0}$ in the space of continuous functions. According to Theorem B.1, we obtain

$$
\begin{aligned}
& \mathbb{E}\left|D_{\rho}(t, x)-D_{\rho}(s, x)\right|^{m} \\
= & \frac{\mathbb{E}\left|D^{\gamma} L\left(t_{0}+\rho t, \rho^{H\left(t_{0}\right)} x+B\left(t_{0}\right)\right)-D^{\gamma} L\left(t_{0}+s \rho, \rho^{H\left(t_{0}\right)} x+B\left(t_{0}\right)\right)\right|^{m}}{\rho^{\left(1-(1+\gamma) H\left(t_{0}\right)\right) m}} \\
\leqslant & C \frac{|t \rho-s \rho|^{\left(1-(1+\gamma) H\left(t_{0}\right)\right) m}}{\rho^{\left(1-(1+\gamma) H\left(t_{0}\right)\right) m}} \leqslant C|t-s|^{m\left(1-H\left(t_{0}\right)(1+\gamma)\right)} .
\end{aligned}
$$

Then to prove the tightness, it suffices to take $m>1 /\left(1-H\left(t_{0}\right)(1+\gamma)\right)$.

It now remains to prove the convergence of the finite-dimensional distributions of $D_{\rho}$, as $\rho$ tends to zero, to those of the fractional derivative of local time of the $\mathrm{fBm} B^{H\left(t_{0}\right)}$ with Hurst parameter $H\left(t_{0}\right)$.

We note $C_{\gamma}=\gamma /(2 \pi \Gamma(1-\gamma))$ and $Y^{\rho}\left(t_{0}\right)=\rho^{H\left(t_{0}\right)} x+B\left(t_{0}\right)$. In view of the definition of $D^{\gamma} L$, we get

$$
\begin{aligned}
& D^{\gamma} L\left(t_{0}+t \rho, \cdot\right)\left(Y^{\rho}\left(t_{0}\right)\right)-D^{\gamma} L\left(t_{0}, \cdot\right)\left(Y^{\rho}\left(t_{0}\right)\right) \\
& =C_{\gamma} \int_{0}^{\infty} \int_{\mathbb{R}}^{t_{0}+t \rho} \int_{t_{0}}^{i u B(s)}\left[e^{-i u\left(Y^{\rho}\left(t_{0}\right)+a\right)}-e^{-i u\left(Y^{\rho}\left(t_{0}\right)-a\right)}\right] \frac{1}{a^{1+\gamma}} d a d u d s \\
& =C_{\gamma} \int_{0}^{\infty} \int_{\mathbb{R}}^{t} \int_{0}^{t} e^{i u B\left(\rho r+t_{0}\right)}\left[e^{-i u\left(Y^{\rho}\left(t_{0}\right)+a\right)}-e^{-i u\left(Y^{\rho}\left(t_{0}\right)-a\right)}\right] \frac{1}{a^{1+\gamma}} \rho d a d u d r \\
& =C_{\gamma} \int_{0}^{\infty} \int_{\mathbb{R}}^{t} \int_{0}^{t} \exp \left[i \frac{v}{\rho^{H\left(t_{0}\right)}} B\left(\rho r+t_{0}\right)\right]\left\{\exp \left[-i \frac{v}{\rho^{H\left(t_{0}\right)}}\left(Y^{\rho}\left(t_{0}\right)+a\right)\right]\right. \\
& =C_{\gamma} \int_{0}^{\infty} \int_{\mathbb{R}}^{t} \int_{0}^{t} \exp \left[i v \frac{B\left(\rho r+t_{0}\right)-B\left(t_{0}\right)}{\rho^{H\left(t_{0}\right)}}\right] \underbrace{1-H\left(t_{0}\right)} \frac{\left.\rho^{\rho}\left(t_{0}\right)-a\right)}{a^{1+\gamma}} d a d v d r \\
& \quad \times\left[e^{-i v(x+b)}-e^{-i v(x-b)}\right] \frac{1}{b^{1+\gamma}} \rho^{1-(1+\gamma) H\left(t_{0}\right)} d b d v d r .
\end{aligned}
$$

We have used the change of variables: $s=\rho r+t_{0}, u=v / \rho^{H\left(t_{0}\right)}$ and $b=a / \rho^{H\left(t_{0}\right)}$. Consequently, we have

$$
\begin{aligned}
D_{\rho}(t, x)=C_{\gamma} \int_{0}^{\infty} \int_{\mathbb{R}} \int_{0}^{t} \exp \left[i v \frac{B\left(\rho r+t_{0}\right)-B\left(t_{0}\right)}{\rho^{H\left(t_{0}\right)}}\right] \\
\times\left[e^{-i v(x-b)}-e^{-i v(x+b)}\right] \frac{1}{b^{1+\gamma}} d r d v d b,
\end{aligned}
$$

which is the fractional derivative of local time of the Gaussian process $B^{\rho}$. 
Finally, by using Lemma 5.2 in [8], concerning the continuity of the fractional derivative, we complete the proof of this result.

4.2. Local limit theorems. Limit theorems for fractional derivative of local time were studied by Yamada [21] for Brownian motion, Fitzsimmons and Getoor [10] for symmetric stable process of index $1<\alpha \leqslant 2$ and Shieh [20] for the $\mathrm{fBm}$ with Hurst parameter $H \in(0,1)$ where this last author proved that if $f$ is in the range of fractional derivative transform, i.e., $f=D_{ \pm}^{\gamma} g$ with $g \in \mathcal{C}^{\beta}$ and $\int g(x) d x \neq 0$, then the family of processes,

$$
\left\{\frac{1}{A^{1-(1+\gamma) H}} \int_{0}^{A t} f\left(X_{s}\right) d s, t \geqslant 0\right\},
$$

converges in law, in the space of continuous functions, as $A \rightarrow+\infty$, to the process

$$
\left\{\int g(x) d x D_{ \pm}^{\gamma} L_{t}(0)\right\} .
$$

Ait Ouahra and Eddahbi [2] generalized the result of Fitzsimmons and Getoor [10] in Hölder space and Ait Ouahra and Ouali [3] extended the result of Shieh [20] to Besov space.

The main tool in the proof of all these results was the self-similarity of the process. In this section, by using the LASS property, we investigate local limit theorems for the occupation time of the $\mathrm{mBm}$.

THEOREM 4.1. Suppose $f=D_{ \pm}^{\gamma} g$, where $g \in C^{\beta}$ with compact support for some $\beta$ such that $0<\gamma<\beta<\xi<\min (1,(1-\lambda) /(2 \lambda))$. Under the assumption $\left(\mathcal{H}_{\beta}\right)$, the following convergence in law holds:

$\lim _{A \rightarrow+\infty} \lim _{\rho \rightarrow 0^{+}} \frac{1}{A^{1-(1+\gamma) H\left(t_{0}\right)}} \int_{0}^{A t} f\left(\frac{B\left(\rho s+t_{0}\right)-B\left(t_{0}\right)}{\rho^{H\left(t_{0}\right)}}\right) d s=\int_{\mathbb{R}} g(x) d x D_{\mp}^{\gamma} l_{t}(0)$.

THEOREM 4.2. Suppose $f=D_{ \pm}^{\gamma} g$, where $g \in C^{\beta}$ with compact support for some $\beta$ such that $0<\gamma<\beta<\xi<\min (1,(1-\lambda) /(2 \lambda))$. Under the assumption $\left(\mathcal{H}_{\beta}\right)$, we have

$$
\frac{1}{\psi(\rho)} \int_{t_{0}}^{\rho t+t_{0}} f\left(\frac{B(s)-B\left(t_{0}\right)-\rho^{H\left(t_{0}\right)} y}{\theta(\rho)}\right) d s \underset{\rho \rightarrow 0^{+}}{\stackrel{\mathcal{L}}{\longrightarrow}} \int_{\mathbb{R}} g(x) d x D_{\mp}^{\gamma} l_{t}(y)
$$

with $\int|g(x)||x|^{\xi-\gamma} d x<\infty, \psi(\rho) / \theta(\rho)=\rho^{1-(1+\gamma) H\left(t_{0}\right)}$ and $\theta(\rho) / \rho^{H\left(t_{0}\right)}=o(1)$.

Proof of The ore m 4.1. Combining the LASS property of $\mathrm{mBm}$ and Theorem VI.4.2 in Gihman and Skorohod [12], we obtain

$$
\int_{0}^{A t} f\left(\frac{B\left(s \rho+t_{0}\right)-B\left(t_{0}\right)}{\rho^{H\left(t_{0}\right)}}\right) d s \underset{\rho \rightarrow 0^{+}}{\stackrel{\mathcal{L}}{\longrightarrow}} \int_{0}^{A t} f\left(B^{H\left(t_{0}\right)}(s)\right) d s,
$$


and by using the result of Shieh [20], the family of processes

$$
\left\{\frac{1}{A^{1-(1+\gamma) H\left(t_{0}\right)}} \int_{0}^{A t} f\left(B^{H\left(t_{0}\right)}(s)\right) d s\right\}_{t \geqslant 0}
$$

converges in law to the process

$$
\left\{\int_{\mathbb{R}} g(x) d x D_{\mp}^{\gamma} l_{t}(0)\right\}_{t \geqslant 0} .
$$

Then the theorem is proved.

Proof of Theorem 4.2. We prove only the case $f=D_{+}^{\gamma} g$ since the proof of the other case is similar. We put $A_{\rho}=\theta(\rho) x+\rho^{H\left(t_{0}\right)} y+B\left(t_{0}\right)$ and $B_{\rho}=\rho^{H\left(t_{0}\right)} y+B\left(t_{0}\right)$. By the occupation time formula and the switching identity, we have

$$
\begin{aligned}
\frac{1}{\psi(\rho)} \int_{t_{0}}^{\rho t+t_{0}} D_{+}^{\gamma} g\left(\frac{B(s)-B\left(t_{0}\right)-\rho^{H\left(t_{0}\right)} y}{\theta(\rho)}\right) d s \\
\quad=\int_{\mathbb{R}} f(x) \frac{L\left(\rho t+t_{0}, A_{\rho}\right)-L\left(t_{0}, A_{\rho}\right)}{\rho^{1-(1+\gamma) H\left(t_{0}\right)}} d x \\
\quad=\int_{\mathbb{R}} g(x) \frac{D_{-}^{\gamma} L\left(\rho t+t_{0}, \cdot\right)\left(A_{\rho}\right)-D_{-}^{\gamma} L\left(t_{0}, \cdot\right)\left(A_{\rho}\right)}{\rho^{1-(1+\gamma) H\left(t_{0}\right)}} d x .
\end{aligned}
$$

Moreover,

$$
\begin{aligned}
& D_{-}^{\gamma} L\left(\rho t+t_{0}, \cdot\right)\left(A_{\rho}\right)-D_{-}^{\gamma} L\left(t_{0}, \cdot\right)\left(A_{\rho}\right) \\
= & D_{-}^{\gamma} L\left(\rho t+t_{0}, \cdot\right)\left(A_{\rho}\right)-D_{-}^{\gamma} L\left(\rho t+t_{0}, \cdot\right)\left(B_{\rho}\right)-D_{-}^{\gamma} L\left(t_{0}, \cdot\right)\left(A_{\rho}\right) \\
- & D_{-}^{\gamma} L\left(t_{0}, \cdot\right)\left(B_{\rho}\right)+D_{-}^{\gamma} L\left(\rho t+t_{0}, \cdot\right)\left(B_{\rho}\right)+D_{-}^{\gamma} L\left(t_{0}, \cdot\right)\left(B_{\rho}\right) \\
= & D_{-}^{\gamma} L\left(\rho t+t_{0}, \cdot\right)\left(B_{\rho}\right)-D_{-}^{\gamma} L\left(t_{0}, \cdot\right)\left(B_{\rho}\right)+D_{-}^{\gamma} L\left(J_{0}, \cdot\right)\left(A_{\rho}\right)-D_{-}^{\gamma} L\left(J_{0}, \cdot\right)\left(B_{\rho}\right)
\end{aligned}
$$

with $J_{0}=\left[t_{0}, t_{0}+\rho t\right]$. Therefore,

$$
\begin{aligned}
\frac{1}{\psi(\rho)} \int_{t_{0}}^{\rho t+t_{0}} D_{+}^{\gamma} g\left(\frac{B(s)-B\left(t_{0}\right)-\rho^{H\left(t_{0}\right)} y}{\theta(\rho)}\right) d s \\
=\frac{D_{-}^{\gamma} L\left(\rho t+t_{0}, \cdot\right)\left(B_{\rho}\right)-D_{-}^{\gamma} L\left(t_{0}, \cdot\right)\left(B_{\rho}\right)}{\rho^{1-(1+\gamma) H\left(t_{0}\right)}} \int_{\mathbb{R}} g(x) d x \\
\\
-\int_{\mathbb{R}} \frac{D_{-}^{\gamma} L\left(J_{0}, \cdot\right)\left(B_{\rho}\right)-D_{-}^{\gamma} L\left(J_{0}, \cdot\right)\left(A_{\rho}\right)}{\rho^{1-(1+\gamma) H\left(t_{0}\right)}} g(x) d x \\
=:(*)-(* *) .
\end{aligned}
$$


By Proposition 4.1 we have $(*) \stackrel{\mathcal{L}}{\rightarrow} D^{\gamma} l_{t}(y) \int_{\mathbb{R}} g(x) d x$ as $\rho \rightarrow 0$. It only remains to prove that $(* *)$ converges to zero. By the Hölder inequality we have

$$
\begin{aligned}
\mathbb{E}\left|\int_{\mathbb{R}} \frac{D_{-}^{\gamma} L\left(J_{0}, \cdot\right)\left(B_{\rho}\right)-D_{-}^{\gamma} L\left(J_{0}, \cdot\right)\left(A_{\rho}\right)}{\rho^{1-(1+\gamma) H\left(t_{0}\right)}} g(x) d x\right| \\
\quad \leqslant \int_{\mathbb{R}}|g(x)||| \frac{D_{-}^{\gamma} L\left(J_{0}, \cdot\right)\left(B_{\rho}\right)-D_{-}^{\gamma} L\left(J_{0}, \cdot\right)\left(A_{\rho}\right)}{\rho^{1-(1+\gamma) H\left(t_{0}\right)}} \|_{2} d x .
\end{aligned}
$$

Applying Lemma 2.1 for the process $B(t)-B\left(t_{0}\right)$ instead of $\mathrm{mBm}$, we have

$$
\begin{aligned}
& \left\|\frac{D_{-}^{\gamma} L\left(J_{0}, \cdot\right)\left(B_{\rho}\right)-D_{-}^{\gamma} L\left(J_{0}, \cdot\right)\left(A_{\rho}\right)}{\rho^{1-(1+\gamma) H\left(t_{0}\right)}}\right\|_{2} \\
& \quad \leqslant C(t \rho)^{1-H\left(t_{0}\right)(1+\xi)}|x|^{\xi-\gamma} \frac{\theta(\rho)^{\xi-\gamma}}{\rho^{1-(1+\gamma) H\left(t_{0}\right)}} \\
& \quad \leqslant C t^{1-H\left(t_{0}\right)(1+\xi)}|x|^{\xi-\gamma}\left(\frac{\theta(\rho)}{\rho^{H\left(t_{0}\right)}}\right)^{\xi-\gamma}
\end{aligned}
$$

for sufficiently small $\rho$ and $0<\gamma<\xi<\min (1,(1-\lambda) /(2 \lambda))$. Hence, (4.2) is dominated by

$$
C t^{1-H\left(t_{0}\right)(1+\xi)} \int_{\mathbb{R}}|g(x)||x|^{\xi-\gamma} d x\left(\frac{\theta(\rho)}{\rho^{H\left(t_{0}\right)}}\right)^{\xi-\gamma}
$$

This last integral is finite by the assumption of the theorem and then $(* *)$ tends to zero as $\rho$ tends to zero.

\section{APPENDIX}

Pro of of Le $\mathrm{m} \mathrm{ma}$ 4.D. To prove the convergence in law, we proceed in two steps. First we prove the tightness of the family $\left\{Y_{\rho}(t, x), t \in[0,1]\right\}_{\rho>0}$ in the space of continuous functions. By using (2.2) and (2.3), for $\rho$ small enough, we obtain

$$
\begin{aligned}
\mathbb{E} \mid Y_{\rho}( & t, x)-\left.Y_{\rho}(s, x)\right|^{m} \\
\quad & \frac{\mathbb{E}\left[L\left(t_{0}+\rho t, \rho^{H\left(t_{0}\right)} x+B\left(t_{0}\right)\right)-L\left(t_{0}+\rho s, \rho^{H\left(t_{0}\right)} x+B\left(t_{0}\right)\right)\right]}{\rho^{\left(1-H\left(t_{0}\right)\right) m}} \\
& \leqslant C_{m}|t-s|^{\left(1-H\left(t_{0}\right)\right) m} .
\end{aligned}
$$

We can take $m>1 /\left(1-H\left(t_{0}\right)\right)$, to get the tightness. 
Now, we prove the convergence of the finite-dimensional distributions of $Y_{\rho}$, as $\rho$ tends to zero, to those of the local time $\ell$ of the $\mathrm{fBm} B^{H\left(t_{0}\right)}$ with Hurst parameter $H\left(t_{0}\right)$. We need to show that, for any $d \geqslant 1, a_{1}, \ldots, a_{d} \in \mathbb{R}$ and $t_{1}, \ldots, t_{d} \in$ $[0,1]$, the following convergence holds:

$$
\sum_{j=1}^{d} a_{j} Y_{\rho}\left(t_{j}, x\right) \stackrel{\mathcal{L}}{\longrightarrow} \sum_{j=1}^{d} a_{j} \ell\left(t_{j}, x\right) \quad \text { as } \rho \rightarrow 0 .
$$

We will show the convergence of the corresponding characteristic function. More precisely, we prove that

$$
\left|\mathbb{E} \exp \left[i \lambda \sum_{j=1}^{d} a_{j} Y_{\rho}\left(t_{j}, x\right)\right]-\mathbb{E} \exp \left[i \lambda \sum_{j=1}^{d} a_{j} \ell\left(t_{j}, x\right)\right]\right| \rightarrow 0 \quad \text { as } \rho \rightarrow 0 .
$$

Let us introduce the following notation:

$$
\begin{gathered}
B^{\rho}(t)=\frac{B\left(t_{0}+\rho t\right)-B\left(t_{0}\right)}{\rho^{H\left(t_{0}\right)}}, \\
\phi_{\varepsilon, x}(X)(t)=\frac{1}{\varepsilon} \int_{0}^{t} \mathbf{1}_{[x, x+\varepsilon]}(X(s)) d s, \\
I_{1}^{\varepsilon, \rho}=\left|\mathbb{E} \exp \left[i \lambda \sum_{j=1}^{d} a_{j} Y_{\rho}\left(t_{j}, x\right)\right]-\mathbb{E} \exp \left[i \lambda \sum_{j=1}^{d} a_{j} \phi_{\varepsilon, x}\left(B^{\rho}\right)\left(t_{j}\right)\right]\right|, \\
I_{2}^{\varepsilon, \rho}=\left|\mathbb{E} \exp \left[i \lambda \sum_{j=1}^{d} a_{j} \phi_{\varepsilon, x}\left(B^{H\left(t_{0}\right)}\right)\left(t_{j}\right)\right]-\mathbb{E} \exp \left[i \lambda \sum_{j=1}^{d} a_{j} \phi_{\varepsilon, x}\left(B^{\rho}\right)\left(t_{j}\right)\right]\right|
\end{gathered}
$$

and

$$
I_{3}^{\varepsilon, \rho}=\left|\mathbb{E} \exp \left[i \lambda \sum_{j=1}^{d} a_{j} \phi_{\varepsilon, x}\left(B^{H\left(t_{0}\right)}\right)\left(t_{j}\right)\right]-\mathbb{E} \exp \left[i \lambda \sum_{j=1}^{d} a_{j} \ell\left(t_{j}, x\right)\right]\right| .
$$

Therefore,

$$
\left|\mathbb{E} \exp \left[i \lambda \sum_{j=1}^{d} a_{j} Y_{\rho}\left(t_{j}, x\right)\right]-\mathbb{E} \exp \left[i \lambda \sum_{j=1}^{d} a_{j} \ell\left(t_{j}, x\right)\right]\right| \leqslant I_{1}^{\varepsilon, \rho}+I_{2}^{\varepsilon, \rho}+I_{3}^{\varepsilon, \rho} .
$$

On the other hand, $Y_{\rho}$ is the local time of $B^{\rho}$ and by using the mean value theorem and the occupation density formula we obtain

$$
\begin{aligned}
I_{1}^{\varepsilon, \rho} & \leqslant C \max _{1 \leqslant j \leqslant d} \mathbb{E}\left|Y_{\rho}\left(t_{j}, x\right)-\phi_{\varepsilon, x}\left(B^{\rho}\right)\left(t_{j}\right)\right| \\
& =C \max _{1 \leqslant j \leqslant d} \mathbb{E}\left|\frac{1}{\varepsilon} \int_{x}^{x+\varepsilon} Y_{\rho}\left(t_{j}, y\right) d y-Y_{\rho}\left(t_{j}, x\right)\right| \\
& \leqslant C \max _{1 \leqslant j \leqslant d} \frac{1}{\varepsilon} \int_{x}^{x+\varepsilon}\left\|Y_{\rho}\left(t_{j}, y\right)-Y_{\rho}\left(t_{j}, x\right)\right\|_{L^{m}(\Omega)} d y
\end{aligned}
$$


and

$$
\begin{aligned}
& \left\|Y_{\rho}\left(t_{j}, y\right)-Y_{\rho}\left(t_{j}, x\right)\right\|_{L^{m}(\Omega)} \\
& =\| \frac{L\left(t_{0}+\rho t_{j}, \rho^{H\left(t_{0}\right)} y+B\left(t_{0}\right)\right)-L\left(t_{0}, \rho^{H\left(t_{0}\right)} y+B\left(t_{0}\right)\right)}{\rho^{1-H\left(t_{0}\right)}} \\
& \quad-\frac{L\left(t_{0}+\rho t_{j}, \rho^{H\left(t_{0}\right)} x+B\left(t_{0}\right)\right)-L\left(t_{0}, \rho^{H\left(t_{0}\right)} x+B\left(t_{0}\right)\right)}{\rho^{1-H\left(t_{0}\right)}} \|_{L^{m}(\Omega)} .
\end{aligned}
$$

On the other hand, putting $I_{j}=\left[t_{0}, t_{0}+\rho t_{j}\right]$, we get

$$
\begin{aligned}
& \left\|\frac{L\left(I_{j}, \rho^{H\left(t_{0}\right)} y+B\left(t_{0}\right)\right)-L\left(I_{j}, \rho^{H\left(t_{0}\right)} x+B\left(t_{0}\right)\right)}{\rho^{1-H\left(t_{0}\right)}}\right\|_{L^{m}(\Omega)} \\
\leqslant & \frac{C_{m}\left(\rho t_{j}\right)^{1-H\left(t_{0}\right)(1+\xi)} \rho^{H\left(t_{0}\right) \xi}|x-y|^{\xi}}{\rho^{1-H\left(t_{0}\right)}} \leqslant \frac{C_{m} t_{j}^{1-H\left(t_{0}\right)(1+\xi)} \rho^{1-H\left(t_{0}\right)}|x-y|^{\xi}}{\rho^{1-H\left(t_{0}\right)}} \\
\leqslant & C_{m}|x-y|^{\xi} .
\end{aligned}
$$

Therefore, (5.2) is dominated by

$$
C_{m} \max _{1 \leqslant j \leqslant d} \frac{1}{\epsilon} \int_{x}^{x+\varepsilon}|x-y|^{\xi} d y=C_{m} \varepsilon^{\xi}
$$

Then (5.2) converges to zero as $\varepsilon$ tends to zero uniformly in $\rho$.

We deal now with $I_{2}^{\varepsilon, \rho}$. Since the family of processes $B^{\rho}(t), t \in[0,1]_{\rho>0}$, converges in distribution to the $\mathrm{fBm} B^{H\left(t_{0}\right)}(t), t \in[0,1]$, with Hurst parameter $H\left(t_{0}\right)$, the second term converges to zero as $\rho$ tends to zero, by Lemma 5.1 in Boufoussi et al. [7]. The last term in (5.1) is treated in a similar way to the first one and the proof of the finite-dimensional convergence is complete.

Acknowledgments. We would like to thank Professors Marco Dozzi and Brahim Boufoussi for their constructive suggestions. We are also grateful to the referee for making thoughtful comments which have led to several improvements in the paper, especially in the proof of Theorem B.2.

\section{REFERENCES}

[1] M. Ait Ouahra, Weak convergence to fractional Brownian motion in some anisotropic Besov space, Ann. Math. Blaise Pascal 11 (1) (2004), pp. 1-17.

[2] M. Ait Ouahra and M. Eddahbi, Théorèmes limites pour certaines fonctionnelles associées aux processus stables sur l'espace de Hölder, Publ. Mat. 45 (2) (2001), pp. 371-386.

[3] M. Ait Ouahra and M. Ouali, Occupation time problems for fractional Brownian motion and some other self-similar processes, Random Oper. Stoch. Equ. 17 (1) (2009), pp. 69-89.

[4] A. Benassi, S. Jaffard, and D. Roux, Elliptic Gaussian random processes, Rev. Mat. Iberoam. 13 (1) (1997), pp. 19-90. 
[5] S. M. Berman, Local nondeterminism and local times of Gaussian processes, Indiana Univ. Math. J. 23 (1973/74), pp. 69-94.

[6] B. Boufoussi, M. Dozzi, and R. Guerbaz, On the local time of multifractional Brownian motion, Stochastics 78 (1) (2006), pp. 33-49.

[7] B. Boufoussi, M. Dozzi, and R. Guerbaz, Sample path properties of the local time of multifractional Brownian motion, Bernoulli 13 (3) (2007), pp. 849-867.

[8] M. Eddahbi and J. Vives, Chaotic expansion and smoothness of some functionals of the fractional Brownian motion, J. Math. Kyoto Univ. 43 (2) (2003), pp. 349-368.

[9] H. Ezawa, J. R. Klauder, and L. A. Shepp, Vestigial effects of singular potentials in diffusion theory and quantum mechanics, J. Math. Phys. 16 (1975), pp. 783-799.

[10] P. J. Fitzsimmons and R. K. Getoor, Limit theorems and variation properties for fractional derivatives of the local time of a stable process, Ann. Inst. H. Poincaré Probab. Statist. 28 (2) (1992), pp. 311-333.

[11] M. Fukushima, A decomposition of additive functionals of finite energy, Nagoya Math. J. 74 (1979), pp. 137-168.

[12] I. I. Gîhman and A. V. Skorohod, The Theory of Stochastic Processes. I, Springer, New York-Heidelberg 1974.

[13] G. H. Hardy and J. E. Littlewood, Some properties of fractional integrals. I, Math. Z. 27 (1) (1928), pp. 565-606.

[14] M. Jolis and N. Viles, Continuity in law with respect to the Hurst parameter of the local time of the fractional Brownian motion, J. Theoret. Probab. 20 (2) (2007), pp. 133-152.

[15] J. Lévy-Véhel, Introduction to the multifractal analysis of images, in: Fractal Image Encoding and Analysis, Y. Fisher (Ed.), Springer, New York 1995.

[16] J. Lévy-Véhel and R. F. Peltier, Multifractional Brownian motion: Definition and preliminary results, [Research Report], RR-2645, INRIA, 1995.

[17] M. Meerschaert, D. Wu, and Y. Xiao, Local times of multifractional Brownian sheets, Bernoulli 14 (3) (2008), pp. 865-898.

[18] B. Pesquet-Popescu and J. Lévy-Véhel, Stochastic fractal models for image processing, IEEE Signal Processing Magazine 19 (5) (2002), pp. 48-62.

[19] S. G. Samko, A. A. Kilbas, and O. I. Marichev, Fractional Integrals and Derivatives: Theory and Applications, Gordon and Breach Science Publishers, Yverdon 1993.

[20] N. Shieh, Limit theorems for local times of fractional Brownian motions and some other selfsimilar processes, J. Math. Kyoto Univ. 36 (4) (1996), pp. 641-652.

[21] T. Yamada, On some limit theorems for occupation times of one-dimensional Brownian motion and its continuous additive functionals locally of zero energy, J. Math. Kyoto Univ. 26 (2) (1986), pp. 309-322.

Mohamed Ait Ouahra

Laboratoire de Modélisation

Stochastique et Déterministe et URAC (04)

Faculté des Sciences Oujda, Maroc

E-mail: ouahra@gmail.com

Hanae Ouahhabi

Department of Statistics

College of Business and Economics

United Arab Emirates University

E-mail: ahanae31@gmail.com
Raby Guerbaz Laboratoire MAEGE and Department SMAEG FSJES Ain Sebaa Hassan II University of Casablanca, Morocco E-mail:rguerbaz@gmail.com

Aissa Sghir

Laboratoire de Modélisation Stochastique et Déterministe Département de Mathématiques Faculté des Sciences Oujda Université Mohammed Premier, Maroc E-mail: sghir.aissa@gmail.com

Received on 22.6.2017;

revised version on 27.9.2017 